\title{
FACTORES ASOCIADOS A CONOCIMIENTOS SOBRE PREVENCIÓN DEL CÁNCER GÁSTRICO EN PACIENTES EN UN HOSPITAL DE LIMA - PERÚ, 2017
}

\author{
FACTORS ASSOCIATED WITH KNOWLEDGE ON THE PREVENTION OF GASTRIC CANCER IN PATIENTS AT \\ A HOSPITAL OF LIMA - PERU, 2017 \\ Mariela Berrospi-Zavalala, , Sussan D. Llocclla-Delgado 2,b, Lucy E. Correa-López ${ }^{3}$
}

\begin{abstract}
RESUMEN
Objetivo: Determinar los factores asociados a conocimientos sobre la prevención del cáncer gástrico en pacientes atendidos en consultorio externo del Hospital Vitarte de Septiembre - Noviembre del 2017. Métodos: Estudio transversal, analítico y observacional. La muestra estuvo conformada por 321 pacientes quienes cumplieron con los criterios de selección. Se aplicó el test de nivel de conocimientos sobre prevención del cáncer gástrico y el test de Graffar. Para el análisis de los datos se empleó el software estadístico IBM SPSS versión 23. Resultados: El 62\% de la población encuestada obtuvo nivel de conocimiento medio. Además, la asociación del nivel de conocimiento, la situación laboral, el acceso $a$ internet y el estrato socioeconómico fue estadísticamente significativa ( $p=0.019, p=0.018, p=0.001)$. Conclusión: Se concluye que el nivel de conocimiento bajo y medio sobre prevención de cáncer gástrico se asoció significativamente con no tener ocupación, no tener acceso a internet y pertenecer a un nivel socioeconómico IV y V.
\end{abstract}

Palabras clave: Cáncer gástrico; Prevención; Estrato socioeconómico; Conocimiento. (fuente: DeCS BIREME)

\begin{abstract}
Objective: To determine the factors associated with knowledge on the prevention of gastric cancer in patients attended at the external office of the Vitarte Hospital in September - November 2017. Methods: Cross-sectional, analytical and observational study. The sample consisted of 321 patients who met the selection criteria. The knowledge level test on gastric cancer prevention and the Graffar test was applied. The statistical software IBM SPSS version 23 was used to analyze the data. Results: $62 \%$ of the surveyed population obtained a medium level of knowledge. In addition, the association of the level of knowledge, work situation, access to the internet and the socioeconomic stratum was statistically significant $(p=0.019$, $p=0.018, p=0.001$ ). Conclusion: It is concluded that the level of low and medium knowledge about gastric cancer prevention was significantly associated with having no occupation, not having access to the internet and belonging to a socioeconomic level IV and V.
\end{abstract}

Key words: Gastric cancer; Prevention; Knowledge; Social class. (source: MeSH NLM)

'Hospital de Vitarte, Lima-Perú.

Universidad del Zulia, Maracaibo-Estado Zulia, Venezuela.

${ }^{3}$ Instituto de Investigación en Ciencias Biomédicas, Universidad Ricardo Palma, Lima-Perú

a Médico Interno.

${ }^{b}$ Médico Cirujano.

Citar como: Mariela Berrospi-Zavala, Sussan D. Llocclla-Delgado, Lucy E. Correa-López. Factores asociados a conocimientos sobre prevención del cáncer gástrico en pacientes en un hospital de Lima-Perú, 2017. [Artículo Original].2019;19(2):00-00. (Abril 2019). DOl 10.25176/RFMH.v19.n2.2064 


\section{INTRODUCCIÓN}

El cáncer gástrico es el segundo cáncer más frecuente del mundo, superado solamente por el cáncer de pulmón'. Posee una alta mortalidad y una variación geográfica significativa² ${ }^{2}$ La variación geográfica es una característica del cáncer gástrico. Los países con más altas tasas ajustadas de mortalidad son: Bulgaria, Colombia, Costa Rica, Chile, China, Islandia, Japón, Portugal y algunos países de la ex URSS. Por el contrario, en países como EE.UU., India, Filipinas y la mayoría de los países africanos esta neoplasia es poco relevante ${ }^{3}$.

La Organización Mundial de la Salud (OMS) menciona que el cáncer gástrico se encuentra dentro de las cinco principales neoplasias más frecuentes en el mundo contemporáneo como causas de defunción a nivel mundial. Es por esto que, el cáncer es un problema creciente en estos países en el que se estima un incremento de la incidencia, llegando a acumular el $70 \%$ de los nuevos casos en $2030^{5,6}$.

En el Perú, según el Instituto Nacional de Enfermedades Neoplásicas (INEN), la incidencia anual del cáncer es aproximadamente 150.7 casos por cada 100,000 habitantes, con un promedio de 45,000 nuevos casos, siendo el cáncer gástrico una de las entidades más agresivas y frecuentes, representando a nivel nacional el $11.1 \%$ de las causas de mortalidad por cáncer en varones y $8.6 \%$ en mujeres ${ }^{5,8}$. A pesar de su importancia, el cáncer gástrico recibe poca atención de las agencias de financiación de la investigación o de las organizaciones de salud pública. Por otro lado, el Instituto Nacional del Cáncer anualmente gasta aproximadamente $\$ 12$ millones en programas directamente relacionados con el cáncer gástrico, solo el $0.2 \%$ de su presupuesto, y solo el $10 \%$ de esta cantidad se destina a la investigación de prevención ${ }^{9}$.

En Latinoamérica, el nivel de conocimiento sobre prevención del cáncer gástrico es en general bajo, según los pocos estudios realizados, así como el de Doval, donde encuentra que, en los estudiantes de bachillerato de Bogotá, Colombia; el nivel de conocimientos sobre los factores de riesgo para el cáncer era bajo ${ }^{10,12}$. Asimismo, en Perú, un estudio realizado en choferes en Lima, se encontró un nivel pobre sobre los conocimientos de prevención de cáncer gástrico; de la misma forma con estudiantes de enfermería en Huánuco ${ }^{15,17}$.

Esto nos hace inferir que el nivel de conocimientos sobre prevención del cáncer gástrico en la población de nuestro país es bajo; sin embargo, aún no existen suficientes estudios para poder afirmarlo. Por lo tanto, el objetivo principal del presente estudio fue determinar los factores asociados a conocimientos sobre la prevención del cáncer gástrico en pacientes atendidos en consultorio externo del Hospital Vitarte durante el período Septiembre - Noviembre del 2017.

\section{MÉTODOS}

Se realizó un estudio de tipo transversal, analítico y observacional. La población estuvo conformada por 321 pacientes quienes cumplieron con los criterios de inclusión y exclusión. Se analizaron 7 variables, de los cuales el nivel de conocimiento fue la variable dependiente, se evaluó la asociación entre esta variable con la edad, sexo, nivel socioeconómico, grado de instrucción, estado de ocupación y acceso a internet.

La técnica de recolección de datos fue mediante el test de nivel de conocimientos sobre prevención del cáncer gástrico y el test de Graffar.

Para el análisis de los datos se empleó la estadística analítica usando el programa Microsoft Excel 2013 para MS Windows y el software estadístico IBM SPSS versión 23.

\section{RESULTADOS}

De acuerdo con el análisis descriptivo de nuestra población encuestada, encontramos que, el $28.97 \%$ (93) se encuentra entre los 31 a 40 años de edad, el 65\% (209) pertenecieron al sexo femenino, el 62.62\% (201) tenían grado de instrucción nivel secundaria. Asimismo, el 54\% (174) no laboraban, el 70\% (224) no tenían acceso a internet, el 51.1\% (164) pertenecían al IV nivel socioeconómico; y sólo el 3.1\% (10) de los pacientes atendidos en consultorio externo del hospital Vitarte pertenecían al V nivel socioeconómico. Tabla 1. 
Tabla 1. Características generales de los pacientes del consultorio externo del Hospital Vitarte.

\begin{tabular}{|c|c|c|c|}
\hline VARIABLES SC & GRÁFICAS & RECUENTO & $\%$ \\
\hline \multirow{2}{*}{ Sexo } & Masculino & 112 & $35 \%$ \\
\hline & Femenino & 209 & $65 \%$ \\
\hline \multirow{5}{*}{ Edad } & 18-30 años & 55 & $17.13 \%$ \\
\hline & $31-40$ años & 93 & $28.97 \%$ \\
\hline & 41-50 años & 90 & $28.04 \%$ \\
\hline & $51-60$ años & 62 & $19.31 \%$ \\
\hline & $>61$ años & 21 & $6.54 \%$ \\
\hline \multirow{3}{*}{ Grado de instrucción } & Primaria & 62 & $19.31 \%$ \\
\hline & Secundaria & 201 & $62.62 \%$ \\
\hline & Superior & 58 & $18.07 \%$ \\
\hline \multirow{2}{*}{ Situación laboral } & Labora & 147 & $46 \%$ \\
\hline & No labora & 174 & $54 \%$ \\
\hline \multirow{2}{*}{ Internet } & Acceso & 97 & $30 \%$ \\
\hline & No acceso & 224 & $70 \%$ \\
\hline \multirow{4}{*}{ Nivel socioeconómico } & II & 18 & $5.60 \%$ \\
\hline & III & 129 & $40.20 \%$ \\
\hline & IV & 164 & $51.10 \%$ \\
\hline & V & 10 & $3.10 \%$ \\
\hline
\end{tabular}

En cuanto al nivel de conocimiento en la tabla 2 , se de conocimiento alto, mientras que, $1 \%$ nivel de observa que el $62 \%$ de los pacientes presentaron un conocimiento bajo. nivel de conocimiento medio, el $37 \%$ con un nivel

Tabla 2. Nivel de conocimiento de los pacientes sobre prevención de cáncer gástrico.

\begin{tabular}{ccc}
\hline CONOCIMIENTO & $\mathbf{N}^{\circ}$ & $\%$ \\
\hline Bajo & 3 & $1 \%$ \\
Medio & 200 & $62 \%$ \\
Alto & 118 & $37 \%$ \\
Total & 321 & $100 \%$ \\
\hline
\end{tabular}

En la tabla 3, se observa que, de los pacientes con un nivel de conocimiento medio y bajo (203), el $49.26 \%$ (100) no laboraban, mientras que, de los 118 pacientes con un conocimiento alto, el $62.71 \%$ (74) también estaban sin laborar, siendo esta relación estadísticamente significativa $(p=0.019)$. Asimismo, se evidencio que de los pacientes con un nivel de conocimiento medio y bajo (203), el 74.38\% (151) no tenían acceso a internet, mientras que, de los 118 con un conocimiento alto, el $61.86 \%(73)$ tampoco tenían acceso a internet $(p=0.018)$. Finalmente, se encontró que de los 203 pacientes con un conocimiento medio y bajo, el $63.55 \%$ (129) pertenecían a un estrato socioeconómico IV / V frente al $38.14 \%$ (45) de los pacientes con un conocimiento alto $(p=0.001)$. 
Tabla 3. Análisis bivariado del nivel de conocimiento sobre prevención del cáncer gástrico y las variables sociodemográficas.

NIVEL DE CONOCIMIENTO

BAJO/MEDIO ALTO

OR IC $95 \%$

p

\begin{tabular}{|c|c|c|c|c|c|c|}
\hline Edad & $\begin{array}{l}<40 \text { años } \\
>40 \text { años }\end{array}$ & $\begin{array}{l}95 \\
108\end{array}$ & $\begin{array}{l}45 \\
73\end{array}$ & 1.42 & $0.87-2.33$ & 0.131 \\
\hline \multirow[b]{2}{*}{ Sexo } & Femenino & 126 & 83 & \multirow[b]{2}{*}{0.69} & \multirow[b]{2}{*}{$0.41-1.15$} & \multirow[b]{2}{*}{0.133} \\
\hline & Masculino & 77 & 35 & & & \\
\hline Grado de & No superior & 171 & 92 & \multirow{2}{*}{1.51} & \multirow{2}{*}{$0.81-2.79$} & \multirow{2}{*}{0.159} \\
\hline instrucción & Superior & 32 & 26 & & & \\
\hline \multirow{2}{*}{ Situación laboral } & No Labora & 100 & 74 & \multirow{2}{*}{0.57} & \multirow{2}{*}{$0.35-0.94$} & \multirow{2}{*}{0.019} \\
\hline & Labora & 103 & 44 & & & \\
\hline \multirow{2}{*}{ Acceso a internet } & Sin acceso & 151 & 73 & \multirow{2}{*}{1.79} & \multirow{2}{*}{$1.06-2.99$} & \multirow{2}{*}{0.018} \\
\hline & Con acceso & 52 & 45 & & & \\
\hline \multirow{2}{*}{$\begin{array}{l}\text { Estado } \\
\text { socioeconómico }\end{array}$} & IV-V & 129 & 45 & \multirow{2}{*}{2.82} & \multirow{2}{*}{$1.72-4.64$} & \multirow{2}{*}{0.001} \\
\hline & II-III & 74 & 73 & & & \\
\hline
\end{tabular}

Fuente: Encuesta aplicada a los pacientes en el consultorio externo del Hospital Vitarte.

\section{DISCUSIÓN}

Partiendo del nivel de conocimientos sobre prevención del cáncer gástrico, el $62.31 \%$ resultó tener un nivel de conocimiento medio, una pequeña proporción (0.93\%) arrojó un nivel de conocimiento bajo y un $36.76 \%$ obtuvo un nivel de conocimiento alto. Estos resultados difieren un poco del estudio realizado por Cutipa titulado "Nivel de conocimientos sobre prevención de cáncer de estómago en usuarios del C.S Primavera en el distrito El agustino, 2010"15 en el que se observó que del $100 \%$ (72) de usuarios encuestados, el 54\% (39) obtuvo un nivel de conocimiento medio, 31\%(22) obtuvo un nivel de conocimiento bajo y un $15 \%(11)$ un nivel conocimiento alto, concluyendo que el nivel de conocimientos de esos usuarios es medio con tendencia a bajo.

Según la asociación de nuestras variables más representativas encontradas en esta investigación, se demuestra a través del análisis de información de datos de los entrevistados que, de aquellos que obtuvieron un nivel de conocimiento medio y bajo no contaban con trabajo (49.26\%) frente al $62.71 \%$ que obtuvieron un nivel de conocimiento alto, obteniendo una asociación estadísticamente significativa $(p=0.019)$. Esto puede explicarse debido a que la población desempleada al tener menos productividad en su vida cotidiana, adoptan actitudes pasivas pudiéndose volver pocos conscientes de la importancia de su salud ya que podría verse involucrados factores como la depresión al no obtener los ingresos que espera disminuyendo así las proyecciones de su futuro. Lo que acarrearía problemas alimenticios, trastornos del sueño, hábitos nocivos, entre otros ${ }^{19}$.

Por otra parte, tener acceso a internet, facilita tener todo tipo de conocimientos de manera rápida y factible, por ende, permite que los usuarios tengan acceso a áreas de conocimiento diferentes a las que encuentran en su entorno cotidiano, permitiendo así adquirir nuevos conocimientos y/o actualizarse de los mismos y tomando acciones al respecto. En nuestro estudio el $74.38 \%$ de los pacientes encuestados que obtuvieron un nivel de conocimiento medio y bajo no tenían acceso a internet frente al $61.86 \%$ de aquellos que obtuvieron un nivel de conocimiento alto, existiendo una relación estadísticamente significativa 
( $p=0.018$ ). Esto podría decir que, las personas que no tienen internet tienen menos conocimientos debido a que gran parte de la información que se puede obtener sobre prevención del cáncer gástrico proviene del internet; entonces las personas que no tienen internet están con menos posibilidades de poder obtener información actualizada de esta enfermedad. Esta investigación coincide con el trabajo realizado por Sandy Karyna Martinez Poves, titulado "Uso del internet y su influencia en los conocimientos y actitudes sobre el embarazo, en gestantes atendidas en el instituto nacional materno perinatal durante el año 2014"24 donde concluyó que el uso del internet influyó en el conocimiento $(p<0.001)$ y las actitudes $(p=0.002)$ sobre el embarazo, en las gestantes atendidas en el Instituto Nacional Materno Perinatal durante el año 2014, puesto que en el grupo que hizo uso del internet se encontró conocimiento "Alto" y actitud "Favorable", en mayor porcentaje, comparado con las que no hicieron uso de internet.

Por último, se cree que el nivel socioeconómico representa un rol importante en las desigualdades en salud. Los pocos ingresos podrían conllevar a tener una limitada o no contar con una cobertura de seguro médico lo que influiría en el acceso a la detección temprana y/o tratamiento de las enfermedades que pudieran presentarse en la vida. Estas poblaciones de menor recurso pueden tener acceso limitado a una nutrición saludable conduciendo a comportamientos de riesgo.

En nuestro estudio, asociando el estrato socioeconómico a tener un nivel de conocimiento bajo, medio o alto según los resultados obtenidos, se vio que el $63.55 \%$ de la población que obtuvieron un nivel de conocimiento medio y bajo se encontraban en un estrato socioeconómico IV y $\mathrm{V}$ frente al $38.14 \%$ de aquellos que obtuvieron un nivel de conocimiento alto, siendo esta asociación estadísticamente significativa $(p=0.001)$. Esto podría explicarse en el sentido de que esta población estudiada representa los niveles más bajos del estrato socioeconómico, calificados por tener una condición precaria y/o con incapacidad para cubrir sus necesidades básicas, haciendo prioritario la búsqueda por el salario diario para subsistir descuidando su salud, a pesar de que el Ministerio de salud del país pone a su disposición programas como el Aseguramiento
Universal en Salud para su atención, esta población no acude o lo hace solo cuando ya es demasiado tarde. A su vez, estas condiciones de vivienda en las que habitan, muchas de ellas con servicios sanitarios deficientes, espacios muy reducidos, alimentación no adecuada, entre otras características propician la aparición de enfermedades, como el cáncer gástrico. Nuestro estudio comparte similitud con la investigación hecha por Ramiro Caballero Hoyos y Alberto Villaseñor Sierra, titulado "El estrato socioeconómico como factor predictor del uso constante de condón en adolescentes" 25 donde concluyen que el estrato socioeconómico es un importante factor predictor del uso consistente del condón. Desde esa perspectiva, un nivel socioeconómico bajo podría llevar a conductas de riesgo para enfermedades.

Se recomienda que se refuerce el nivel de conocimientos de la población a fin de concientizar sobre las correctas medidas de prevención del cáncer gástrico para así evitar su desarrollo ya que muchas veces es de clínica silenciosa, para poder así disminuir la incidencia y/o prevalencia del cáncer gástrico.

\section{CONCLUSIÓN}

Se concluye que el nivel de conocimiento bajo y medio sobre prevención de cáncer gástrico se asoció significativamente con no tener ocupación, no tener acceso a internet y pertenecer a un nivel socioeconómico IV y V.

Contribuciones de autoría: Los autores participaron en la génesis de la idea, diseño de proyecto, recolección e interpretación de datos, análisis de resultados, preparación del manuscrito, presente trabajo de investigación.

Financiamiento: Autofinanciado.

Conflicto de interés: Los autores declaran no tener conflicto de interés en la publicación de este artículo.

Recibido: 18 de noviembre de 2018

Aprobado: 25 de enero de 2019

Correspondencia: Mariela Berrospi Zavala

Dirección: Av. Coronel Portillo 382. San Isidro, Lima, Perú.

Teléfono: +51957320530

Correo:berrospi_mariel@hotmail.com 


\section{REFERENCIAS BIBLIOGRÁFICAS}

1. Biffi R., Luca F. Surgical outcome after docetaxel-based neoadjuvant chemotherapy in locally-advanced gastric cancer. World J Gastroenterol, 16 (2010), pp. 868-874

2. Pisani P., Parkin D., Bray F., Ferlay J. Estimates of the Worldwide Mortality From 25 Cancers in 1990 Int. j. Cancer, 83 (1999), pp. 18-29

3. Kamangar F., Dores G., Anderson W. Patterns of Cancer Incidence, Mortality, andPrevalence Across Five Continents: Defining Priorities to Reduce Cancer Disparities inDifferent Geographic Regions of the World J. Clin. Oncology, 24 (2006), pp. 2137-2150

4. Verdalet Olmedo, M. Factores Asociados al Desarrollo de Cáncer Gástrico en Pacientes del CECan. Tesis para obtener el grado de Maestra en Salud Pública. Universidad Veracruzana, Veratruz, México, 2009.

5. Herrero R, Parsonnet J, Greenberg ER. Prevention of Gastric Cancer. JAMA. 2014;312(12):1197-1198.

6. Martinich Seguich, M. Sobrevida de pacientes con cáncer gástrico atendidos en el Hospital Regional de la ciudad de Punta Arenas. Tesis para optar al grado de Magister en Salud Pública, Chile, 2015.

7. López Cañizares, L., Machuca Serrano, T. y Maldonado Espinoza, M. Los factores de riesgo asociados a cáncer gástrico en pacientes diagnosticados desde 2000 al 2010 en el Instituto del Cáncer, Solca - Cuenca. Tesis previa a la obtención del título de médico y médica, Universidad de Cuenca, Ecuador, 2012.

8. Solís Alcívar, D. Incidencia y perfil clínico de cáncer gástrico en pacientes atendidos en el Área de Gastroenterología en el Hospital de especialidades Abel Gilbert Pontón en el periodo de enero 2012 a diciembre 2014. Tesis para optar el título de médico. Universidad de Guayaquil, Ecuador, 2015.

9. Pérez Rodríguez, S. y Rodríguez Rubinos, R., González Hernández, A y Álvarez Delgado, M. en su estudio sobre Caracterización del cáncer gástrico en pacientes del municipio Caimito. Centro Nacional de Toxicología. La Habana. Cuba, 2012.

10. Lancheros Galvis, N. Factores alimentarios y nutricionales asociados al cáncer gástrico y presencia de helicobacter pylori a nivel mundial 1995-2013: Revisión del Estado del Arte. Tesis para optar al título de Nutricionista Dietista. Pontificia Universidad Javeriana, Bogotá Colombia, 2013.

11. Zambrano Plata, G., Flórez Villamizar, M., Lozano Avendaño, L. y Rubio Meneses, Y. Prevalencia de los factores de riesgo para cáncer gástrico en universitarios. Universidad de Francisco de Paula Santander. Colombia, 2014.

12. Ulloa Meza, F. y Molina Marín, L. Factores de riesgo de cáncer gástrico en pacientes diagnosticados en el Departamento de Patología del "Hospital San Juan de Dios, de Estelí, durante enero de 2010 a agosto de 2012. Tesis para título de doctor en medicina y cirugía. Universidad Nacional Autónoma de Nicaragua, León, Nicaragua, 2013.

13. Daza Duque, D. Cáncer gástrico en Colombia entre 2000 y 2009 Universidad del Rosario - Universidad CES, Bogotá, Colombia, 2012.
14. Rondán Guerrero, F. Factores de riesgo asociados al cáncer gástrico en pacientes atendidos en el consultorio externo del servicio de gastroenterología del HNHU durante el año 2014. Tesis para optar el título profesional de médico cirujano. Universidad Ricardo Palma, Perú, 2016.

15. Cutipa Clemente, K. Nivel de conocimientos sobre prevención de cáncer de estómago en usuarios del C.S. Primavera en el distrito EI Agustino, 2010. Tesis para optar por el Título Profesional de Licenciada en enfermería. Universidad Nacional Mayor de San Marcos, Lima, Perú, 2010.

16. Villanueva Rivera, M. Conocimientos y actitudes sobre prevención del cáncer de estómago en estudiantes de enfermería de la Universidad de Huánuco, 2015. Tesis para optar el título profesional de licenciada en enfermería. Universidad de Huanuco, Perú, 2016.

17. Campos Guzmán, R. Tratamiento quirúrgico y análisis de sobrevida del cáncer gástrico avanzado. Tesis para optar grado académico de maestría en medicina con mención en cirugía general. Universidad de San Martín de Porres, Lima, Perú, 2013.

18. Rivera Romaní, J. Complicaciones de la gastrectomía por cáncer gástrico Hospital Nacional Alberto Sabogal Sologuren 2010 - 2012, Tesis para optar el título de especialista en cirugía general. Universidad de San Martín de Porres, Lima, Perú, 2015.

19. Sánchez Arias, C., Características sociodemográficas, hábitos de consumo alimentario y sustancias nocivas en pobladores con diagnóstico de gastritis y que acuden al centro de Salud de Quilmaná - Cañete 2014. Tesis para optar el título de licenciado en enfermería. Universidad de San Martín de Porres, Lima, Perú, 2014.

20. Paul Pilco, S., Nazario Ortiz, C., Néstor Juárez, K., Edwin Velásquez, I. Cáncer gástrico en un hospital general: Hospital Santa Rosa. Revista de Gastroenterología del Perú, Lima, Perú, 2009.

21. Reinhold E. teoría del conocimiento [Internet]. Ciencias filosóficas; [citado 2015 marzo 23], disponible en: http://www.mercaba.org/ Filosofia/PDF/Tor\%C3\%Ada\%20del\%20Conocimiento.pdf

22. Trejo Martínez F. Aplicación del Modelo de Nola Pender a un Adolescente con Sedentarismo.2010.[fecha de acceso 25 de setiembre];9(1).URL disponible en: http://www.medigraphic.com/pdfs/ enfneu/ene-2010/ene101j.pdf

23. Ramírez A. y Sánchez R., Helicobacter pylori y cáncer gástrico. Rev. gastroenterol. Perú [online]. 2008, vol.28, n.3 [citado 2015-04-02], pp. 258-266.

24. Martinez Poves, Sandy Karyna, [Tesis] "Uso del internet y su influencia en los conocimientos y actitudes sobre el embarazo, en gestantes atendidas en el instituto nacional materno perinatal durante el año 2014". Universidad Nacional Mayor de San Marcos. Disponible en: http://cybertesis.unmsm.edu.pe/handle/cybertesis/4166

25. Ramiro Caballero Hoyos y Alberto Villaseñor Sierra. El estrato socioeconómico como factor predictor del uso constante de condón en adolescentes. Revist Saúde Pública. São Paulo - SP - Brazil. Rev Saúde Pública 2001;35(6):531-8 [online]. Disponible en: https://www.scielosp. org/scielo.php?script=sci_arttext\&pid=S0034-89102001000600006 